\title{
ADE Regimen
}

National Cancer Institute

\section{Source}

National Cancer Institute. ADERegimen. NCI Thesaurus. Code C9579.

A regimen consisting of cytarabine (Ara-C), daunorubicin and etoposide, that is used as an induction treatment for pediatric acute myeloid leukemia (AML). 